University of Louisville

ThinkIR: The University of Louisville's Institutional Repository

Faculty Scholarship

4-2017

\title{
Can the hopelessness model of depression and the response style theory be integrated?
}

\author{
Patrick Pössel \\ University of Louisville \\ Stephanie Winkeljohn Black \\ University of Louisville
}

Follow this and additional works at: https://ir.library.louisville.edu/faculty

Part of the Counseling Psychology Commons

\section{Original Publication Information}

This is the peer reviewed version of the following article:

Pössel, Patrick and Stephanie Winkeljohn Black. "Can the Hopelessness Model of Depression and the Response Style Theory be integrated?" 2017. Journal of Counseling and Development 95(2): 180-191. which has been published in final form at: https://doi.org/10.1002/jcad.12130

This article may be used for non-commercial purposes in accordance with Wiley Terms and Conditions for Self-Archiving.

This Article is brought to you for free and open access by ThinkIR: The University of Louisville's Institutional Repository. It has been accepted for inclusion in Faculty Scholarship by an authorized administrator of ThinkIR: The University of Louisville's Institutional Repository. For more information, please contact thinkir@louisville.edu. 
Can the Hopelessness Model of Depression and the Response Style Theory be Integrated?

Patrick Pössel \& Stephanie Winkeljohn Black

Department of Counseling and Human Development, University of Louisville, Louisville, KY, USA

Corresponding author:

Patrick Pössel, Dr. rer. soc.

Department of Counseling and Human Development

University of Louisville

2301 S. Third Street

Louisville, KY 40292

USA

+1-(502)852-0623 (office)

$+1-(502) 852-0629($ fax $)$

e-mail: patrick.possel@louisville.edu 


\begin{abstract}
The hopelessness model (Abramson et al., 1989) and response style theory (Nolen-Hoeksema et al., 1992) have been integrated in various ways, but these integrations have not been compared. German college students $(N=311$; mean age $=23.27$ years, $S D=6.57$ years, $80 \%$ female $)$ rated their depressive symptoms, negative inferences, and rumination three times. Findings supported an integrated model where individual inferences predict and interact with the rumination subtype brooding to affect depressive symptoms.
\end{abstract}

Keywords: depression; hopelessness model; response style theory; rumination; adults. 
Two major cognitive theories to explain the development and maintenance of depression are the hopelessness model (Abramson, Alloy, \& Metalsky, 1989) and the Response Style Theory (RST; Nolen-Hoeksema, Girgus, \& Seligman, 1992). The empirically supported (see for reviews Abramson et al., 2002; Thomsen, 2006) models offer a theoretical rationale for which constructs contribute to the onset and maintenance of depression and they are associated with effective interventions (e.g., Hawley et al., 2014; Michalak, Hölz, \& Teismann, 2011). Thus, an empirically-supported theoretical model integrating both theories and describing how the variables proposed in one model relate to the variables of the other model would provide a theoretical framework for how therapeutic techniques from one cognitive model can influence constructs from another model. Moreover, an integrative model may allow increased effectiveness of cognitive psychotherapies for depressive symptoms as counselors can integrate therapeutic techniques from different cognitive models into one theory-driven treatment approach (e.g., Hawley et al.; Michalak et al.). Thus, the purpose of the present study is to test and compare multiple theoretically possible models to integrate the hopelessness model and the RST into one model to explain the development and maintenance of depression.

\section{Hopelessness Model}

The hopelessness model (Abramson et al., 1989) proposes that negative inferences and hopelessness work in a sequence to cause depressive symptoms. The negative inferences about negative events form what is called a negative cognitive style. Individuals with this style will make negative inferences about a negative event's (1) internality of cause, (2) stability of cause, (3) globality of cause, and (4) consequences, and (5) characteristics of him/herself following the event. Negative inferences about stability, globality, consequences, and characteristics of the self but not inferences about internality are proposed to lead to hopelessness (Abramson et al., 
1989), which triggers the development of a variety of symptoms of hopelessness depression, a subtype of depression. While negative inferences about internality of cause do not lead to hopelessness, they lead to low self-esteem, another symptom of hopelessness depression. While the different negative inferences are traditionally summarized into a composite score (Haeffel et al., 2008), studies with college students in the Midwestern U.S. (65.2\% female; Haeffel, 2010) and Canadian adults in a clinical sample (86.1\% female; Abela, Aydin, \& Auerbach, 2006) found evidence that more variance of depressive symptoms is explained when considering the individual negative inferences. In other words, the individual negative inferences and not the composite score should be studied as the later one can mask one very negative inferential style because the other inferences are less negative. While empirically well supported (see for a review Abramson et al., 2002), the inferences proposed in the hopelessness model do not completely explain the development and maintenance of depressive symptoms. Thus, additional predictors should be considered and integrated into the hopelessness model. One possible cognitive construct that it missing in the hopelessness model but that is also related to the development and maintenance of depressive symptoms is how individuals process and respond to their depressive mood (Nolen-Hoeksema et al., 1992).

\section{Response Style Theory}

The RST asserts that the individual's cognitive response to his/her depressive mood determines the onset, severity, and length of a depressive episode (Nolen-Hoeksema et al., 1992). Individuals who react to a depressive mood by repetitively thinking about their mood and the consequences of the mood are said to have a ruminative response style, which magnifies his/her depressive mood. This ruminative response style can be divided into three subtypes: Brooding, reflection, and depression-related rumination (Treynor, Gonzalez, \& Nolen-Hoeksema, 2003). 
However, the authors noted that the depression-related subtype simply reflects depressive symptoms, rather than representing a ruminative response style, and suggested to focus on the two other ruminative subtypes. Brooding involves moody and passive thinking about one's actions or situation (e.g., thinking about how a situation could have gone differently), whereas reflection has a neutral valence and involves a problem-solving approach (e.g., analyzing why events make one feel a certain way) with the goal of contemplation and understanding one's depressive mood. Despite positive correlations between brooding and reflection, only brooding was found to be a consistent predictor of depressive symptoms in college students and outpatients treated for depression in Hong Kong (77\% female; Lo, Ho, \& Hollon, 2008) in Western U.S. adults (53.5\% female; Treynor et al.), in German adolescents (34.7\% female; Winkeljohn Black \& Pössel, 2013) and in Southern U.S. adolescents (63.9\% female; Winkeljohn Black \& Pössel, 2015). The situation regarding reflection is less consistent as some have found no association with depressive symptoms (Lo et al.; Winkeljohn Black \& Pössel) while others found significant associations between reflection and depressive symptoms (Treynor et al.). Integrating the Hopelessness Model and the Response Style Theory

Similarities between both models and associations between negative inferences outlined in the hopelessness model (Abramson et al., 1989) and RST (Nolen-Hoeksema et al., 1992) are obvious. One of apparent similarity between both cognitive models is their classification as cognitive vulnerability-stress models. This implies that the interactions between cognitive vulnerabilities and activating negative events are used to explain why some individuals develop depression while others do not. Beyond this relatively crude classification, studies found rumination predicts hopelessness (Lavender \& Watkins, 2004) and (Lyubomirsky \& NolenHoeksema, 1995 [second study reported]) and even that hopelessness mediates the association 
between rumination and depressive symptoms (Sarin, Abela, \& Auerbach, 2005). Finally, the authors themselves have pointed out different possible associations between negative inferences and rumination (Abramson et al., 1989; Alloy et al., 2000). However, neither of the models includes both negative inferences and rumination. Thus, a theory-driven and empirically supported model integrating the hopelessness model and the RST is needed.

One suggested combination of both cognitive vulnerability models is a moderation model (Alloy et al., 2000; Ciesla \& Roberts, 2007; Figure 1, top). Alloy et al. suggested that individuals who have negative inferences and ruminate about these inferences are more likely to develop depressive symptoms than individuals who have only one or neither of these cognitive vulnerabilities. Ciesla and Roberts expanded on this proposition by outlining that rumination might affect the influence of negative inferences by bringing them to mind more often. Supporting this moderation model, Alloy and colleagues found in their cross-sectional study that individuals with a cognitive risk (a combination of a composite of negative inferences about stability, globality, consequences, and the self and dysfunctional attitudes; Beck, 1976) and a ruminative response style were more likely to have a history of major depression than individuals with only one or neither of these cognitive vulnerabilities. However, two experimental (Ciesla \& Roberts, [second and third studies reported]) and two longitudinal studies did not find empirical support for this moderation model (Ciesla, Felton, \& Roberts, 2011; Robinson \& Alloy, 2003). Despite Alloy and colleagues' (2000) support for the moderation model, their study has some limitations. First, the authors used retrospective data. Second, Alloy et al. used cognitive risk, a combination of negative inference style and dysfunctional attitudes - a construct from Beck's theory (1976) of depression. Third, rumination but not its subtypes brooding and reflection were examined. While two longitudinal studies (Pössel, 2011; Winkeljohn Black \& 
Pössel, 2015) did not find support for the influence of an interaction between dysfunctional attitudes and either of the ruminative subtypes on depressive symptoms, another study found support for the influence of a dysfunctional attitudes by brooding interaction (Winkeljohn Black \& Pössel, 2013). Thus, it is possible that the significant effect of the interaction between cognitive risk and rumination in Alloy et al.'s (2000) study was not related to the negative inferences but to dysfunctional attitudes.

Ciesla's laboratory (Ciesla \& Roberts, 2007 [second and third study reported]) and longitudinal studie (Ciesla et al., 2011) overcame the limitations in Alloy et al.'s (2000) study. However, neither of their studies found support for the attribution style by rumination (brooding and reflection) interaction predicting depressive symptoms. However, Ciesla and colleagues (Ciesla \& Roberts; Ciesla et al.) measured only attribution style (composite of negative inferences about internality, stability, and globality). Thus, it is unclear if either or both ruminative subtypes are moderators in the associations between some or all of the individual negative inferences and depressive symptoms. Further, it is unclear if rumination or its subtypes moderate the associations between all or only some of the negative inferences outlined in the hopelessness theory.

Another proposed model to integrate negative inferences (Abramson et al., 1989) and rumination (Nolen-Hoeksema et al., 1992) is a mediation model (Figure 1, middle). Abramson et al. stated that individuals experiencing hopelessness might ruminate, leading to attention and sleep problems. Thus, rumination may be a mediator between negative inferences and depressive symptoms. Three studies support this mediation hypothesis. In their longitudinal study Spasojević and Alloy (2001) found that rumination fully mediated the positive association between negative inferences (measured as a composite score of negative inferences about 
stability, globality, consequences, and the self) and future depressive episodes. Similarly, in the two cross-sectional studies Lo et al. (2008) found that brooding partially mediated the positive association between attribution style (negative inferences about internality, stability, and globality) and self-reported depressive symptoms. Moreover, reflection did not mediate the association between attribution style and depressive symptoms in either of Lo et al.'s samples.

In sum, there is empirical support for rumination, particularly the brooding subtype, as mediator between negative inferences and depressive symptoms. However, it is unclear whether rumination is especially important for specific individual negative inferences (i.e., stability, globality, internality, consequences, and characteristics of the self). As negative inferences about stability, globality, consequences, and characteristics of the self but not inferences about internality are proposed to lead to hopelessness (Abramson et al., 1989), one could speculate that internality inferences are not associated with rumination. However, Lo et al. (2008) included internality inferences alongside the other four inferences in their study and found that rumination only partially mediated the association between negative inferences and depressive symptoms. In contrast, Spasojević and Alloy's (2001) study did not include internality inferences and they found rumination fully mediated the association between negative inferences and depressive symptoms. Thus, while one might speculate about differential associations between the individual negative inferences and rumination, no study has evaluated rumination (or brooding, specifically) as mediator in the association between individual negative inferences and depressive symptoms yet. In addition, only cross-sectional studies (e.g., Lo et al.) have researched brooding and reflection as separate mediators alongside constructs from the hopelessness theory. Thus, based on the inconsistent literature on reflection described above (Lo et al.; Treynor et al., 2003; Winkeljohn Black \& Pössel, 2013, 2015) it is unclear as to whether 
brooding and reflection, rather than brooding alone, would mediate longitudinal associations between some or all of the individual negative inference and depressive symptoms.

A final possible integrated model we suggest involves including both the proposed mediation and moderation models (Alloy et al., 2000). In this combined integrative model, each individual negative inference predicts brooding and/or reflection while also interacting with rumination to predict depressive symptoms. This model is not only consistent with the theoretical considerations to integrate the hopelessness model with the RST (Nolen-Hoeksema et al., 1992), it also would explain how both the mediation and moderation integrated models have been supported in the literature consistent with empirical studies supporting both of the suggested integrated models (Alloy et al.; Lo et al., 2008; Spasojević \& Alloy. 2001). So far, however, no study has tested this combined integrative model (Figure 1, bottom).

\section{Current Study}

Summarized, three different models - mediation, moderation, combined - have been proposed to integrate negative inferences (hopelessness model; Abramson et al., 1989) with rumination as described in the RST (Alloy et al., 2000; Lo et al., 2008; Nolen-Hoeksema et al., 1992; Spasojević \& Alloy, 2001). The mediation and moderation model were empirically examined in multiple studies resulting in the above outlined conflicting findings. Thus, we hypothesized a combined integrative model in which brooding would be predicted by all individual negative inferences (mediation) and would interact with the individual negative inferences (moderation) to predict depressive symptoms. Based on the fact that reflection is either not included in many of the previous studies attempting to integrate the hopelessness and the RST associations between missing or when it was included, the literature about the association between reflection and depressive symptoms is inconsistent (Lo et al.; Treynor et al., 
2003; Winkeljohn Black \& Pössel, 2013, 2015) it seemed crucial to include reflection in the study. Thus, it could not be determined $a^{\prime}$ priori whether reflection would be predicted by the individual negative inferences (mediation) and would interact with the individual negative inferences (moderation) to predict depressive symptoms. Further, we proposed that all individual negative inferences (stability, globality, consequences, and self) except inferences about internality would predict rumination (mediation) and interact with rumination (moderation) to predict depressive symptoms. Summarized the core research questions is which of the three proposed integrative models (i.e., mediation, moderation, combined) best describes the development and maintenance of depressive symptoms and whether reflection plays a role in this model or not.

\section{Methods}

\section{Participants}

Participants $(N=398$, mean age $=23.27$ years, $\mathrm{SD}=6.57$ years, age range: 18 to 52 years, $80 \%$ female) were German college students. From waves $1-3,87$ students (66 females) dropped out. There were no differences between the dropouts and remaining students in sex $\left(\chi^{2}(1)=1.13, p=.287\right)$ or depressive symptoms $(t(387)=-0.69, p=.494)$. However, dropouts were significantly older $(t(396)=-2.02, p<.05)$. Thus, the final sample used for analyses included 311 participants.

\section{Measures}

Depressive Symptoms. The Center for Epidemiological Studies - Depression Scale (CES - D; Radloff, 1977) consists of 20 items (e.g., "During the past week, there were things that upset me that usually do not upset me.’); developed to be as a quickly administered, economic screening instrument to measure depressive symptoms based on self-report. 
Frequency of symptoms is rated on a four-point scale, with a higher sum of the item endorsements indicating higher frequency of occurrence. The internal consistency and criterion validity of the CES-D in the standardization sample was good $(\alpha=.85 ; r=.56$ with clinician's ratings of depression, respectively).

Negative Inferences. The Cognitive Style Questionnaire (CSQ; Haeffel et al., 2008) measures inferences about causes, consequences, and the self in relation to negative events (Abramson et al., 1989) with 24 hypothetical event scenarios (12 negative and 12 positive). Only the negative event scenarios were used in this study. Respondents were presented with a hypothetical event and asked to write down one cause for the event. Respondents then rated the degree to which the cause of the hypothetical event was (a) internal, (b) stable, and (c) global (negative inferences about the causes of negative events). Next, they rated the likelihood that further negative consequences would result from the event (negative inferences about consequences). Finally, they rated the degree to which the occurrence of the event meant that the self is flawed (negative inferences about the self). Each rating uses a 7-point Likert scale, with higher summed scores representing more negative inferences. Haeffel and colleagues found CSQ scale scores to have good internal consistency (ranging .83-.91) and criterion validity with the BDI-II $(r=.37)$.

Brooding and Reflection. The Rumination Response Subscale (RRS) of the Response Styles Questionnaire (RSQ; Nolen-Hoeksema \& Morrow, 1991) consists of 18 4-point Likert items that measure how often a participant engaged in various behaviors in response to depressed mood. The RRS can be divided into the subscales brooding and reflection as well as items that measure depression-related cognitions (Treynor et al., 2003). In this study, only the brooding (e.g., "I think ,Why do I always react this way?"”) and reflection (e.g., "I analyze recent events 
to try to understand why I am depressed.") subscales were included, as the depression-related subscale is often regarded as a measure of depressive symptoms and not as a measure of rumination distinctly separate from depression. Higher summed scores in each subscale represent more engagement in those specific ruminative behaviors. Treynor et al. found $\alpha$ 's of .77 (brooding) and .72 (reflection), as well as high correlations between depressive symptoms (BDI-II) and brooding $(r=.44)$ and low correlations between depressive symptoms and reflection $(r=.12)$, as expected from the literature.

\section{Procedures}

Participants were recruited from a participant pool of a psychology undergraduate program. While the participation in a certain number of studies was a program requirement, the participation in the particular study was not. Thus, the data were collected using convenience sampling. Participants completed questionnaire batteries in groups of 8 to 15 at the beginning (wave 1), middle (wave 2), and end (wave 3) of the fall semester at 4-week intervals. At wave 1, all individual negative inferences, both response styles (brooding and reflection), and depressive symptoms were measured. At wave 2, only brooding and reflection were assessed, and at wave 3, depressive symptoms were measured. Within each wave, the order of the questionnaires was counterbalanced across the sample following the Latin square design. To be able to connect data from the different waves while keeping confidentiality, the participants developed their own code based on the initial of their first name, their last name, and their date of birth. Informed consent was obtained and each participant received course credit for participation. The ethical committee of the German Psychological Association approved this study.

\section{Data Analysis}


Full information maximum likelihood (FIML) - which estimates a likelihood function for each individual based on the variables that are present so that all the available data are used - was used, enabling the inclusion of participants with missing data to compensate for missing data related to attrition. FIML is a robust estimator even if data are not missing at random (Collins, Schafer, \& Cam, 2001; Graham, 2003). This is of crucial importance for this study as data at later waves are not missing at random, as participants that dropped out being older than participants who remained in the study.

To test which model fit the data best, Cole and Maxwell's (2003) approach for multiwave studies using structural equation modeling was used. The analyses were conducted with IBM AMOS 21 to calculate path models (Arbuckle, 1999). Goodness of fit of the models was tested with $\chi^{2}$. However, as $\chi^{2}$ is known to increase with sample size and degrees of freedom, the $\chi^{2}$ was complemented by the root mean squared of the residuals (RMSEA; Steiger $\&$ Lind, 1980), Tucker-Lewis Index (TLI; Tucker \& Lewis, 1973), and Comparative Fit Index (CFI; Bentler, 1990). While a full explanation of these indices and their limitations is beyond the scope of this article, a short description seems necessary. Statistically nonsignificant values of $\chi^{2}$ indicate a good fit of the model to the data. An RMSEA value of .00 indicates a perfect model fit; a value of $\leq .05$ is conventionally regarded as an indicator of a good model fit; and a value of $\leq .08$ is seen as acceptable (Hu \& Bentler, 1999). TLI and CFI values of $\geq .95$ indicate a good model fit and values of $\geq .90$ are regarded as acceptable (Hu \& Bentler).

Two different tests were used to compare models. First, $\Delta$ CFI was calculated by subtracting the CFI value of one model from the CFI value of another model. When $\Delta \mathrm{CFI}$ is > .002 the model with higher CFI fits the data significantly better. When $\Delta \mathrm{CFI}$ is $\leq .002$ both models fit equally well from a statistical point of view and the more parsimonious model (more 
$d f$ s) should be accepted (Meade, Johnson, \& Braddy, 2008). Second, nested models (i.e., models with the same number of observed variables) were compared by subtracting the $\chi^{2}$ values as well as the $d f$ s of the models from each other ( $\chi^{2}$ difference tests). When $\Delta \chi^{2}$ is significant for $\Delta d f$, the models are seen as significantly different from each other. To estimate if and how much an integration of the hopelessness model (Abramson et al., 1989) and the RST (Nolen-Hoeksema et al., 1992) increases the predictive value of the cognitive constructs of both theories, percentage of explained variance in depressive symptoms was calculated for each model.

To test the hypothesized mediations, a temporal relation must exist between the independent (e.g., inferences), mediation (brooding \& reflection), and dependent variables (depressive symptoms) (Stice, Rohde, Seeley, \& Gau, 2010): that is, the mediators must be measured temporally after the independent variables and before the dependent variable. In order to test for multiple mediators, $95 \%$ bootstrapping confidence intervals (CIs) were calculated using the bias-corrected percentile method (Preacher \& Hayes, 2008). Based on this approach, multiple mediation effects exist when the indirect effect (i.e., the effect from independent variable through all possible mediators to dependent variable) is significant.

To allow for the calculation of moderations, all variables were z-standardized. To calculate the moderation variables, the $\mathrm{z}$-standardized scores of the variables that were predicted to interact (e.g., inference about internality at wave $1 \&$ brooding at wave 1 ) were multiplied with each other and these moderation variables (inference about internality by brooding at wave 1) were entered along with the z-standardized scores of the original variables. All analyses were calculated using both the full CES-D and only the CES-D items that measure hopelessness depression (excluded items: 4, 10, 15, 16, 18, and 19). As both analyses revealed the same pattern of results, only the findings with the full CES-D are presented here. 


\section{Results}

Descriptive data, internal consistency, and correlations for all instruments at all three waves are presented in Table 1. The majority of the measures correlated with each other.

\section{Determination of the Best Fitting Model}

Six different models were tested and compared with each other to identify the model that fit the data best. In all six models the influence of depressive symptoms at wave 1 was controlled for. The goodness of fit indices for five of the six models were good. However, Model 3 had a significant $\chi^{2}$-value, an acceptable CFI, and unacceptable RMSEA.

Overall, the six models can be understood as two sets of models including three models each. The first set of models included the two original models: Model 1 represented the original hopelessness model (Abramson et al., 1989), without brooding and reflection or their interactions with the individual negative inferences $\left(\chi^{2}(1)=.208, p=.648\right.$, CFI (1.0), RMSEA $\left.(0.001)\right)$. Model 2 described the RST (Nolen-Hoeksema et al., 1992) without negative inferences from the hopelessness model or their interactions with response styles $\left(\chi^{2}(1)=.015, p=.901, \mathrm{CFI}(1.0)\right.$, RMSEA (0.001)). Model 3 allowed for direct associations between each individual negative inference and both response styles (brooding and reflection) measured at wave 1 and depressive symptoms measured at wave 3 but did not allow for associations between the response style constructs and constructs of the hopelessness model $\left(\chi^{2}(12)=71.406, p=.001\right.$, CFI (.947), RMSEA (0.112)). The second set of models described different versions of an integrated hopelessness-rumination model. Model 4 was based on Alloy et al.’s (2000) and Ciesla and Roberts' (2007) moderation model $\left(\chi^{2}(3)=1.140, p=.767\right.$, CFI (1.0), RMSEA (0.001)). Thus, this model included the individual negative inferences, brooding and reflection, and the interaction of each of the negative inferences with brooding and reflection at wave 1 as 
predictors of depressive symptoms at wave 3 (Figure 1, top). Model 5 followed the proposal that rumination mediates the association between negative inferences and depressive symptoms. In other words, Model 5 included the individual negative inferences at wave 1 and both response styles at wave 1 and $2\left(\chi^{2}(4)=5.323, p=.256\right.$, CFI (.999), RMSEA (0.029)). Further, the negative inferences were directly and also through the response styles indirectly associated with depressive symptoms at wave 3 (Figure 1, middle). The final model (Model 6) represented the combination the mediation and the moderation model to integrate the hopelessness model with the $\operatorname{RST}\left(\chi^{2}(6)=5.260, p=.511\right.$, CFI (1.0), RMSEA (0.001)). Thus, in this model the individual negative inferences, brooding and reflection, and the interactions between the negative inferences and the response styles at wave 1 were directly and indirectly through brooding and reflection indirectly associated with depressive symptoms at wave 3 (Figure 1, bottom).

First, Models 1-3 (no integrations) were compared with each other. A comparison of Model 1 and Model 3 demonstrated significant differences between the two, $\Delta \mathrm{CFI}=0.053 ; \Delta \chi^{2}$ $(11, N=397)=71.198, p<.001$, favoring Model 1. In addition, Model 1 explained 22.9\% variance in depressive symptoms compared with $21.9 \%$ explained variance in Model 3. Thus, Model 1, which represented the original hopelessness model (Abramson et al., 1989), was retained. Similarly, a comparison of Model 2 (16.2\% explained variance in depressive symptoms) and Model 3 favored Model 2, which represented the original RST (Nolen-Hoeksema et al., 1992), $\Delta \mathrm{CFI}=0.053 ; \Delta \chi^{2}(11, N=397)=71.391, p<.001$.

Second, Models 1 and 2 were compared to the moderation model (Model 4; Figure 1, top). These comparisons found nonsignificant differences: Model 1 vs. Model 4: $\Delta \mathrm{CFI}=0.000$; $\Delta \chi^{2}(2, N=397)=0.932, p=.628 ;$ Model 2 vs. Model 4: $\Delta \mathrm{CFI}=0.000 ; \Delta \chi^{2}(2, N=397)=$ $1.125, p=.570$. Thus, as Model 4 (25.5\% explained variance) had more $d f$ s and therefore was 
more parsimonious, the moderation model was retained. Similarly, a comparison of Models 1 and 2 with the mediation model (Model 5; Figure 1, middle) revealed nonsignificant differences: Model 1 vs. Model 5: $\Delta \mathrm{CFI}=0.001 ; \Delta \chi^{2}(3, N=397)=5.115, p=.164$; Model 2 vs. Model 5: $\Delta \mathrm{CFI}=0.001 ; \Delta \chi^{2}(3, N=397)=5.308, p=.151$. Thus, as the mediation model $(24.3 \%$ explained variance) was more parsimonious (more $d f \mathrm{~s}$ ), Model 5 was retained.

Third, the moderation model (Model 4; Figure 1, top) and the mediation model (Model 5; Figure 1, middle) were compared with the combined integrative model (Model 6; Figure 1, bottom). These comparisons revealed nonsignificant differences, Model 4 vs. Model 6: $\Delta \mathrm{CFI}=$ $0.000 ; \Delta \chi^{2}(3, N=397)=4.120, p=.249 ;$ Model 5 vs. Model 6: $\Delta \mathrm{CFI}=0.001 ; \Delta \chi^{2}(2, N=397)$ $=0.063, p=.969$. Thus, as the combined integrative model (Model 6; 27.6\% explained variance) had more $d f$ s and therefore was more parsimonious, this model was retained. This model explained $4.7 \%$ more variance than the original hopelessness model and $11.4 \%$ more variance than the original RST model.

Finally, an inspection of the associations in the combined integrated model (Model 6) revealed that many of the associations were not statistically significant. Thus, following Burkholder and Harlow's (2003) suggestion, we set all paths with a $p \geq .20$ to zero and calculated a simplified combined integrated model $\left(\right.$ Model 7: $\chi^{2}(32)=16.509, p=.989$, CFI (1.0), RMSEA (0.001)). Further, we compared Model 6 with this simplified Model 7. This comparison revealed a nonsignificant difference, $\Delta \mathrm{CFI}=0.000 ; \Delta \chi^{2}(26, N=397)=11.249, p=$ .995. Thus, as the simplified combined integrative model ( $27.0 \%$ explained variance) had more $d f$ s and therefore was more parsimonious, this model was retained (Figure 2).

\section{Analyses of Individual Associations in the Best-Fitting Model}


As the simplified combined integrative model (Model 7) was retained, it was crucial to test (a) which of the cognitive variables measured at wave 1 were associated with depressive symptoms measured at wave 3 and (b) with of the rumination subtypes served as mediator in these associations. Thus, it is of importance that only brooding at wave 2 but not reflection at wave 2 was significantly associated with depressive symptoms at wave 3 . Thus, all indirect effects were mediated by brooding at wave 2 and none by reflection at wave 2 .

Inferences about the consequences and the self, the interactions of inferences about the internality and stability with brooding, and brooding at wave 1 were mainly marginally significantly and directly associated with depressive symptoms at wave 3 (Table 2). Further, inferences about the self, brooding, and reflection at wave 1 were all significantly and indirectly associated with depressive symptoms at wave 3 (Table 2). Thus, the association between the latter three cognitive variables from both cognitive theories at wave 1 and depressive symptoms at wave 3 were mediated by brooding at wave 2 .

Finally, brooding and reflection at wave 1 predicted not only themselves and each other at wave 2 , but the interaction of inferences about the stability with brooding at wave 1 as well as the interactions of all inferences with reflection at wave 1 predicted reflection at wave 2 . However and as stated above, reflection at wave 2 was not significantly associated with depressive symptoms at wave 3. Thus, while interesting these associations are not relevant for the purpose of the manuscript.

\section{Discussion}

The primary goal of this three-wave longitudinal study was to integrate negative inferences from the hopelessness model (Abramson et al., 1989) and rumination from the RST (Nolen-Hoeksema et al., 1992) into a single model. An integrated model in which rumination 
was predicted by individual negative inferences and interacted with individual inferences to predict depressive symptoms fit the data better than the original cognitive models and the other integrated models. This final integrative model merged two proposals and considered the differential findings on ruminative brooding and reflection, and their associations to depressive symptoms (Lo et al. 2008; Treynor et al., 2003; Winkeljohn Black \& Pössel, 2013, 2015). It was expected that brooding would be predicted by individual negative inferences and would interact with individual inferences to predict depressive symptoms. However, based on the inconsistent literature on the association of reflection with depressive symptoms (Lo et al.; Treynor et al.; Winkeljohn Black \& Pössel), it was unclear whether reflection would be predicted by individual negative inferences and would interact with individual negative inferences to predict depressive symptoms. Further, it was proposed that all individual negative inferences (stability, globality, consequences, and self) except inferences about internality would predict brooding and also interact with brooding to predict depressive symptoms.

This study revealed three important findings with regard to these hypotheses: First, the combined integrated model in which rumination was predicted by individual negative inferences and interacted with individual inferences while affecting depressive symptoms fit the data better than the other tested original and integrated models.

Second, brooding and reflection predicted depressive symptoms eight weeks later. However, while brooding predicted depressive symptoms directly and indirectly, reflection did this only indirectly via brooding. This might explain the consistent results of previous studies finding only brooding predicting depression (Lo et al., 2008; Winkeljohn Black \& Pössel, 2015) or finding both rumination subtypes predicting depression (Treynor et al., 2003; Winkeljohn Black \& Pössel, 2013). Consistent with the hopelessness model (Abramson et al., 1989), the 
individual inferences about consequences and the self, but not about internality, predicted depressive symptoms eight weeks later. However, not consistent with the hopelessness model the main effects of inferences about stability and globality did not predict depressive symptoms. While the lack of a significant association between the main effect of stability and depressive symptoms could be explained by the simultaneous inclusion of the stability by brooding interaction, which significantly predicted depressive symptoms, the lack of a significant association of inferences of globality with depressive symptoms was surprising.

Third, reflection at wave 2 did not mediate the associations between cognitive variables at wave 1 and depressive symptoms at wave 3 while brooding mediated the association of inferences about the self with depressive symptoms. This finding suggests that brooding becomes the vehicle through which an individual with a negative inferential style about the self processes their negative self-view. As brooding is a passive, internal experience it makes sense that it would allow an individual to access internally (self)-oriented judgments. Once these judgments, or inferences, are accessed, the person would experience depressive symptoms.

If replicated, the findings of this study draw a picture of an interesting pattern of relationships between the negative inferences about the self (Abramson et al., 1989) and brooding (Nolen-Hoeksema et al., 1992). Moreover, the differences among individual inferences' associations to brooding and depressive symptoms indicate that future research needs to consistently address inferences individually rather than as a unitary construct.

This study was the first to research the associations between individual negative inference styles and brooding and reflection instead of composites of multiple negative inference styles and the combined ruminative style. However, this study has limitations as well. The sole use of self-report instruments was a limitation of the present study. First, a mono-method bias from 
using the same informant and method for assessing all constructs in this study was likely.

Second, the use of self-report instruments to measure negative inference styles and ruminative styles could be criticized because it is questionable how much insight individuals really have into their own style of thinking (for a review see Scher, Ingram, \& Segal, 2005). Therefore, information processing paradigms might be better suited than self-report questionnaires when measuring process constructs. Thus, future studies would benefit from the use of multiple assessment methods (e.g., self-report questionnaires, interview data to measure depressive symptoms, \& information processing paradigms). Nevertheless, information-processing paradigms have not been developed for either of the measured cognitive constructs in the present study (Gotlib \& Neubauer, 2000), while self-report instruments are readily available for all of these constructs. Therefore, we decided to use these well-established instruments in our studies. Third, the internal consistency of the RSQ Brooding and Reflection subscales were lower than preferred, this limited reliability and consequently limits validity of the measure as well. However, in the validation sample for these subscales, Treynor and associates (2003) also found low coefficients and suggested that the small number of items comprising each subscale and not the quality of the items impact the reliability of scores. Further, the low scores have also been found in American (Ciesla et al., 2011), Chinese (Yang, Ling, Xiao, \& Yao, 2009), and other German (Pössel, 2011) samples; nonetheless the subscales continue to demonstrate adequate psychometrics and assist in studying and understanding the development of depression across adolescent, young adult, and adult samples globally.

Fourth, an issue impacting the generalizability of the findings is the high proportion of female participants in the current study. Thus sex disparity in studies examining associations between cognitive vulnerabilities and depressive symptoms is common, but it means that these 
models should be interpreted as female-driven explanations for the onset and maintenance of depression. This point becomes particularly salient when considering sex differences in prevalence of depressive symptoms and rumination, which prompted the development of the RST (Nolen-Hoeksema \& Morrow, 1991). Some studies found sex differences regarding how these cognitive vulnerabilities interact to predict depressive symptoms (e.g., Pössel, 2011), while others have found no differences (e.g., Ciesla \& Roberts, 2007). Thus, more equivalent representation of male participants is important to clarify where and how these differences exist.

Lastly, both the hopelessness model (Abramson et al., 1989) and the RST (NolenHoeksema et al., 1992) are vulnerability-stress models. Thus, the cognitive vulnerabilities from each model need to be activated by stressors (e.g., life events and/or daily hassles) in order to impact depressive symptoms. As stress was not included in the present study, it is possible that the associations of cognitive variables with depressive symptoms were underestimated. However, the fact that many of the predicted associations were significant seems to point to the limited impact of the failure to include stress in the present study.

The results of this study are especially significant for clinical applications. The identified integrative model provides a theoretical framework for how therapeutic techniques from one cognitive model can influence constructs from another model. Moreover, the findings may increase the effectiveness of cognitive psychotherapies for depressive symptoms; counselors can integrate therapeutic techniques from different cognitive models into one theory-driven treatment approach (e.g., Hawley et al., 2014; Michalak et al., 2011). For example, the finding that brooding mediated and moderated some of the individual inferential styles and even of reflection suggests that clinicians may want to concentrate on reducing brooding in clients with depressive symptoms. If brooding is the vehicle connecting inferential styles and depressive symptoms, 
focusing on brooding in therapy may alleviate clients' depressive symptoms faster. As there is evidence indicating that mindfulness-based treatments successfully target rumination (e.g., Hawley et al.), such as brooding, mindfulness could be seen as the treatment of choice for clients with depression if the present findings can be replicated.

The applicability of the current findings needs to be contextualized within the age and developmental level of the sample. The current sample of college students represents individuals in emerging adulthood, a developmental stage wherein most mental health issues emerge (Kessler et al., 2005). Moreover, some studies found higher rates of depressive symptoms in emerging adults compared to other adult age groups (e.g., Kessler et al., 2010). The current findings therefore provide college and community counselors working with emerging adults, with information about (a) how to integrate various cognitive approaches to treatment and (b) the importance of mindfulness-based interventions to target cognitive constructs contributing to depressed mood. These considerations can be used in individual counseling and can inform college and community self-help resources and psycho-education, as well as college and community-level intervention and prevention programming. While counselors provide treatment to a variety of developmental groups, from childhood through late adulthood, the opportunity to focus on intervention and prevention with this age group can soften how these emerging adults experience the onset and maintenance of depressive symptoms in mid- to late-adulthood. 


\section{References}

Abela, J.R.Z., Aydin, C., \& Auerbach, R.P. (2006). Operationalizing the "vulnerability" and "stress" components of the hopelessness theory of depression: A multi-wave longitudinal study. Behaviour Research and Therapy, 44, 1565-1583. doi: http://dx.doi.org/10.1016/j.brat.2005.11.010.

Abramson, L. Y., Alloy, L. B., \& Metalsky, G. I. (1989). Hopelessness depression: a theorybased subtype of depression. Psychological Bulletin, 96, 358 - 372.

Abramson, L. Y., Alloy, L. B., Hankin, B. L., Haeffel, G. J., MacCoon, D., \& Gibb, B. E. (2002). Cognitive vulnerability-stress models of depression in a self-regulatory and psychological context. In I. H. Gotlib \& C. L. Hammen (Eds.), Handbook of depression and its treatment (pp. 268 - 294). New York, NY: The Guilford Press.

Alloy, L. B., Abramson, L. Y., Hogan, M. E., Whitehouse, W. G., Rose, D. T., Robinson, M. S., Kim, R. S., \& Lapin, J. B. (2000). The Temple-Wisconsin Cognitive Vulnerability to Depression Project: Lifetime history of Axis I psychopathology in individuals at high and low cognitive risk for depression. Journal of Abnormal Psychology, 109, 403 - 418.

Arbuckle, J. L. (1999). Amos user's guide. Chicago, IL: SmallWaters.

Beck, A.T. (1976). Cognitive therapy and the emotional disorders. New York: International Universities Press.

Bentler, P. M. (1990). Comparative fit indexes in structural models. Psychological Bulletin, 107, 238-246.

Burkholder, G. J. \& Harlow, L. L. (2003). An illustration of a longitudinal cross-lagged design for larger structural equation models. Structural Equation Modeling, 10, 465 - 486. 
Ciesla, J. A., Felton, J. W., \& Roberts, J. E. (2011). Testing the cognitive catalyst model of depression: Does rumination amplify the impact of cognitive diatheses in response to stress? Cognition and Emotion, 25, 1349-1357.

Ciesla, J. A. \& Roberts, J. E. (2007). Rumination, negative cognition, and their interactive effects on depressed mood. Emotion, 7, 555 - 565.

Cole, D. A., \& Maxwell, S. E. (2003). Testing mediational models with longitudinal data: Questions and tips in the use of structural equation modeling. Journal of Abnormal Psychology, 112, 558-577.

Collins, L. M, Schafer, J.L., \& Kam, C-M. (2001). A comparison of inclusive and restrictive strategies in modern missing data procedures. Structural Equation Modeling, 6, 330-351.

Gotlib, I. H., \& Neubauer, D. L. (2000). Information-processing approaches to the study of cognitive biases in depression. In S. L. Johnson, A. M. Hayes, T. M. Field, N. Schneiderman, \& P. M. McCabe (Eds.), Stress, coping, and depression (pp. 117 - 142). Mahwah, NJ: Lawrence Erlbaum Associates.

Graham, J.W. (2003). Adding missing-data-relevant variables to FIML-based structural equation models. Structural Equation Modeling, 10, 80-100.

Haeffel, G.J. (2010). Cognitive vulnerability to depressive symptoms in college students: A comparison of traditional, weakest-link, and flexibility options. Cognitive Therapy and Research, 34, 92-98. doi: 10.1007/s10608-008-9224-8

Haeffel, G. J., Gibb, B. E., Metalsky, G. I., Alloy, L. B., Abramson, L. Y., Hankin, B. L. Joiner, T. E., Jr., \& Swendsen, J. D. (2008). Measuring cognitive vulnerability to depression: Development and validation of the cognitive style questionnaire. Clinical Psychology Review, 28, $824-836$. 
Hawley, L. L., Schwartz, D., Bieling, P. J., Irving, J., Corcoran, K., Farb, N. A. S., Anderson, A. K., \& Segal, Z. V. (2014). Mindfulness practice, rumination and clinical outcome in mindfulness-based treatment. Cognitive Therapy and Research, 38, 1-9. doi: 10.1007/s10608-013-9586-4.

Hu, L. \& Bentler, P. M. (1999). Cut-off criteria for fit indexes in covariance structure analysis: Conventional criteria versus new alternatives. Structural Equation Modeling, 6, 1-55.

Kessler, R. C., Birnbaum, H., Bromet, E., Hwang, I., Sampson, N., \& Shahly, V. (2010). Age differences in major depression: Results from the National Comorbidity Survey Replication (NCS-R). Psychological Medicine, 40, 225-237.

Kessler, R. C., Demler, O., Frank, R. G., Olfson, M., Pincus, H. A., Walters, E. E., ... Zaslavsky, A. M. (2005). Prevalence and treatment of mental disorders, 1990 to 2003. New England Medical Journal, 352, 2515-2523.

Lavender, A. \& Watkins, E., (2004). Rumination and future thinking in depression. British Journal of Clinical Psychology, 43, 129-142.

Lyubomirsky, S. \& Nolen-Hoeksema, S. (1995). Effects of self-focused rumination on negative thinking and interpersonal problem solving. Journal of Personality and Social Psychology, 69, 176-190.

Lo, C. S. L., Ho, S. M. Y., \& Hollon, S. D. (2008). The effects of rumination and negative cognitive styles on depression: a mediation analysis. Behaviour Research and Therapy, 46, $587-495$.

Michalak, J., Hölz, A., \& Teismann, T. (2011). Rumination as a predictor of relapse in mindfulness-based cognitive therapy for depression. Psychology and Psychotherapy, 84, 230-236. doi:10.1348/147608310X520166 
Meade, A. W., Johnson, E. C., \& Braddy, P. W. (2008). Power and sensitivity of alternative fit indices in tests of measurement invariance. Journal of Applied Psychology, 93, 568 - 592.

Nolen-Hoeksema, S. (1991). Responses to depression and their effects on the duration of depressive episodes. Journal of Abnormal Psychology, 100, 569 - 582.

Nolen-Hoecksema, S., Girgus, J. S., \& Seligman, M. E. P. (1992). Predictors and consequences of childhood depressive symptoms: A 5-year longitudinal study. Journal of Abnormal Psychology, 101, $405-422$.

Nolen-Hoeksema, S. \& Morrow, J. (1991). A prospective study of depression and posttraumatic stress symptoms after a natural disaster: the 1989 Loma Prieta earthquake. Journal of Personality and Social Psychology, 61, 115 - 121.

Pössel, P. (2011). Can Beck's theory of depression and the response style theory be integrated? Journal of Counseling Psychology, 58, 618-629. doi: 10.1037/a0025092

Preacher, K. J., \& Hayes, A. F. (2008). Asymptotic and resampling strategies for assessing and comparing indirect effects in multiple mediator models. Behavior Research Methods, 40, 879-891.

Radloff, L. S. (1977). The CES-D: A self-report symptom scale to detect depression in the general population. Applied Psychological Measurement, 3, 385 - 401.

Robinson, M. S. \& Alloy, L. B. (2003). Negative cognitive style and stress-reactive rumination interact to predict depression: a prospective study. Cognitive Therapy and Research, 27, 275292.

Sarin, S., Abela, J. R. Z., Auerbach, R. P. (2005). The response styles theory of depression: A thest of specificity and causal mediation. Cognition and Emotion, 19, 751-761. 
Scher, C. D., Ingram, R. E., \& Segal, Z. V. (2005). Cognitive reactivity and vulnerability: Empirical evaluation of construct activation and cognitive diatheses in unipolar depression. Clinical Psychology Review, 25, 487-510.

Spasojević, J. \& Alloy, L. B. (2001). Rumination as a common mechanism relating depressive risk factors to depression. Emotion, 1, $25-37$.

Steiger, J. H. \& Lind, J. M. (1980, May). Statistically based tests for the number of common factors. Paper presented at the Psychometrika Society meeting, Iowa City, Iowa.

Stice, E., Rohde, P., Seeley, J. R., \& Gau, J. M. (2010). Testing mediators of intervention effects in randomized controlled trials: an evaluation of three depression prevention programs. Journal of Consulting and Clinical Psychology, 78, 273 - 280. doi: 10.1037/a0018396

Thomsen, D. K. (2006). The association between rumination and negative affect: a review. Cognition and Emotion, 20, 1216 - 1235. doi: 10.1080/02699930500473533

Treynor, W., Gonzalez, R. \& Nolen-Hoeksema, S. (2003). Rumination reconsidered: a psychometric analysis. Cognitive Therapy and Research, 27, 247 - 259.

Tucker, L. R. \& Lewis, C. (1973). The reliability coefficient for maximum likelihood factor analysis. Psychometrika, 38, 1-10.

Winkeljohn Black, S. \& Pössel, P. (2013). The combined effects of self-referent information processing and ruminative responses on adolescent depression. Journal of Youth and Adolescence, 42, 1145-1154.

Winkeljohn Black, S. \& Pössel, P. (2015). Integrating Beck's cognitive model and the response style theory in an adolescent sample. Journal of Youth and Adolescence, 44, 195-210. 
Yang, J., Ling, Y., Xiao, J., \& Yao, S-G. (2009). The Chinese version of the Ruminative

Responses Scale in high school students: Is it reliable and valid? Chinese Journal of Clinical Psychology, 17, 27-31. 
Table 1

Descriptive Data, Internal Consistencies, and Correlations between All Instruments $(N \geq 302)$

\begin{tabular}{|c|c|c|c|c|c|c|c|c|c|c|c|}
\hline & CES-Dt1 & CES-Dt3 & CSQit1 & CSQgt1 & CSQst1 & CSQcot1 & CSQset1 & RSQbt1 & RSQrt1 & RSQbt2 & RSQrt2 \\
\hline CES-Dt1 & .90 & & & & & & & & & & \\
\hline CES-Dt3 & $.38 * *$ & .91 & & & & & & & & & \\
\hline CSQit1 & $.17 * *$ & $.17 * *$ & .73 & & & & & & & & \\
\hline CSQgt1 & $.32 * *$ & $.31 * *$ & $.40 * *$ & .82 & & & & & & & \\
\hline CSQst1 & $.18 * *$ & $.19 * *$ & $.42 * *$ & $.64 * *$ & .83 & & & & & & \\
\hline CSQcot1 & $.32 * *$ & $.34 * *$ & $.33 * *$ & $.76^{* *}$ & $.52 * *$ & .90 & & & & & \\
\hline CSQset1 & $.37 * *$ & $.38 * *$ & $.40 * *$ & $.60 * *$ & $.43 * *$ & $.65^{* *}$ & .91 & & & & \\
\hline RSQbt1 & $.25^{* *}$ & $.16^{* *}$ & $.20 * *$ & $.26^{* *}$ & $.18 * *$ & $.29 * *$ & $.36^{* *}$ & .60 & & & \\
\hline RSQrt1 & -.01 & .04 & .02 & $.10^{*}$ & $.17 * *$ & .08 & $.15^{* *}$ & $.35^{* *}$ & .68 & & \\
\hline RSQbt2 & $.25 * *$ & $.29 * *$ & $.16^{* *}$ & $.28 * *$ & $.19 * *$ & $.26 * *$ & $.34 * *$ & $.64 * *$ & $.35 * *$ & .67 & \\
\hline RSQrt2 & .06 & .11 & .04 & $.19 * *$ & $.19 * *$ & .11 & $.16^{* *}$ & $.19 * *$ & $.71 * *$ & $.36 * *$ & .73 \\
\hline Mean & 15.59 & 16.51 & 46.64 & 37.38 & 41.20 & 31.16 & 29.61 & 2.47 & 2.58 & 2.33 & 2.45 \\
\hline $\mathrm{SD}$ & 10.01 & 10.15 & 8.42 & 10.34 & 10.41 & 11.35 & 13.24 & 0.76 & 0.62 & 0.75 & 0.63 \\
\hline
\end{tabular}

Note. Values in the diagonal represent Cronbach's Alpha. CES-D = Center for Epidemiological Studies - Depression; CSQ $i=\mathrm{CSQ}$,

negative events internal-external; CSQg = CSQ, negative events general-specific; CSQs = CSQ, stable-unstable; CSQco = CSQ,

negative inference about consequences; CSQse = CSQ, negative inference about the self; RSQb = Response Style Questionnaire,

brooding; RSQr = Response Style Questionnaire, reflection; $\mathrm{t} 1=$ assessment wave $1 ; \mathrm{t} 2$ = assessment wave 2; $\mathrm{t} 3=$ assessment wave 3 .

$* p<.05 ; * * p<.01$. 
Table 2

Total, Direct, and Indirect Effects and their Confidence Intervals testing for Multiple Mediations among Cognitive Constructs at Times 1 and 2 and Depressive Symptoms at Time 3

\begin{tabular}{|c|c|c|c|}
\hline & Effects & Lower CL & Upper CL \\
\hline \multicolumn{4}{|l|}{ Total effects } \\
\hline CSQit1 - CES-Dt3 & 0.000 & -0.841 & 1.155 \\
\hline CSQgt1 - CES-Dt3 & 0.105 & -1.374 & 1.243 \\
\hline CSQst1-CES-Dt3 & 0.000 & -1.057 & 1.034 \\
\hline CSQcot1 - CES-Dt3 & $1.170+$ & 0.076 & 2.220 \\
\hline CSQset1 - CES-Dt3 & $1.864 *$ & 0.926 & 2.886 \\
\hline RSQbt1 - CES-Dt3 & -0.062 & -0.954 & 0.858 \\
\hline RSQrt1 - CES-Dt3 & $0.273^{*}$ & 0.134 & 0.538 \\
\hline CSQit1 by RSQbt1 - CES-Dt3 & $1.226+$ & 0.125 & 2.330 \\
\hline CSQst1 by RSQbt1 - CES-Dt3 & $-1.068 *$ & -1.887 & -0.318 \\
\hline CES-Dt1 - CES-Dt3 & $0.272 *$ & 0.173 & 0.407 \\
\hline RSQbt2 - CES-Dt3 & $1.900^{*}$ & 1.116 & 3.024 \\
\hline \multicolumn{4}{|l|}{ Direct effects } \\
\hline CSQcot1 - CES-Dt3 & $1.170+$ & 0.076 & 2.220 \\
\hline CSQset1 - CES-Dt3 & $1.666+$ & 0.784 & 2.704 \\
\hline RSQbt1 - CES-Dt3 & $-1.101+$ & -2.280 & -0.010 \\
\hline CSQit1 by RSQbt1 - CES-Dt3 & $1.226+$ & 0.125 & 2.330 \\
\hline CSQst1 by RSQbt 1 - CES-Dt3 & $-1.068 *$ & -1.887 & -0.318 \\
\hline CES-Dt1 - CES-Dt3 & $0.272 *$ & 0.173 & 0.407 \\
\hline
\end{tabular}


$\begin{array}{llll}\text { RSQbt2 - CES-Dt3 } 3.900^{*} & 1.116 & 3.024\end{array}$

Indirect effects

$\begin{array}{lccc}\text { CSQgt1 - CES-Dt3 } & 0.105 & -0.093 & 0.330 \\ \text { CSQset1 - CES-Dt3 } & 0.198^{*} & 0.030 & 0.426 \\ \text { RSQbt1 - CES-Dt3 } & 1.039^{*} & 0.532 & 1.790 \\ \text { RSQrt1 - CES-Dt3 } & 0.273^{*} & 0.134 & 0.538\end{array}$

Note. $\mathrm{CL}=$ Confidence limit; $\mathrm{CES}-\mathrm{D}=$ Center for Epidemiological Studies - Depression; CSQi = CSQ, negative events internal-external; CSQg = CSQ, negative events generalspecific; CSQs = CSQ, stable-unstable; CSQco = CSQ, negative inference about consequences; CSQse = CSQ, negative inference about the self; RSQb = Response Style Questionnaire, brooding; RSQr = Response Style Questionnaire, reflection; $\mathrm{t} 1$ = assessment wave $1 ; \mathrm{t} 2=$ assessment wave $2 ; \mathrm{t} 3=$ assessment wave $3 .+p<.10 ; * p<.05$ 


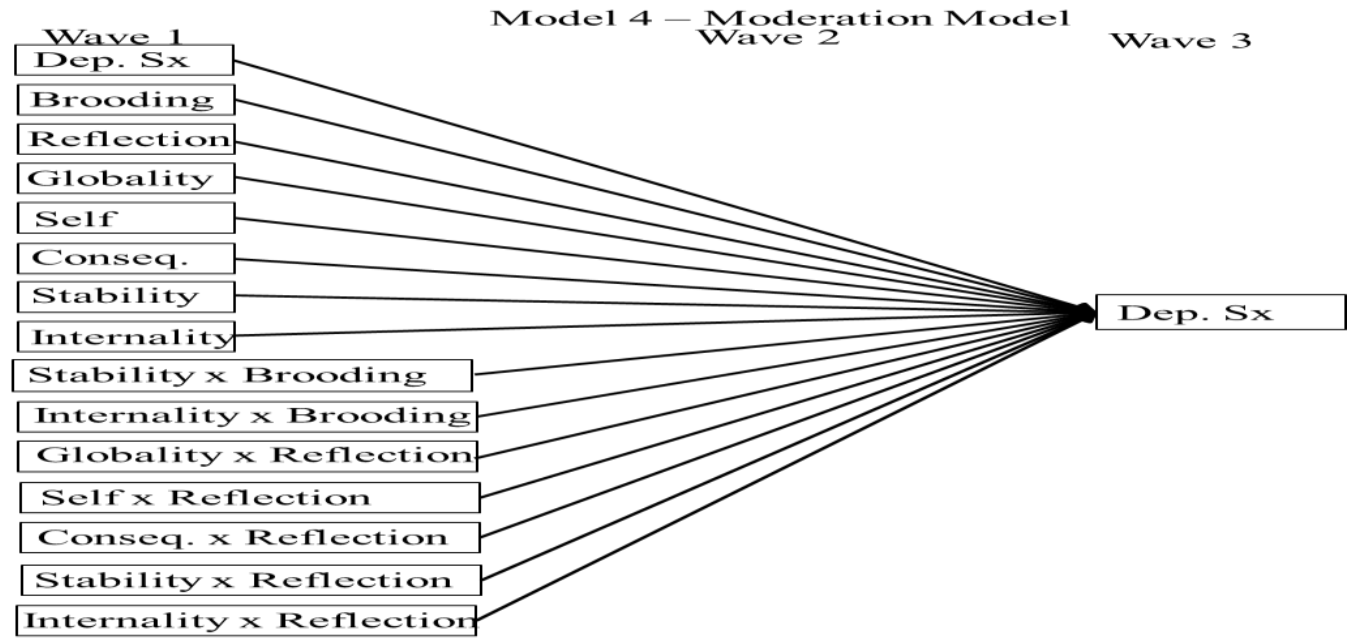

\section{Wave 1}

Dep. Sx

Brooding

Reflection

Globality

Self

Conseq.

Stability

Internality
Model 5-Mediation Model

Wave 2

Wave 3

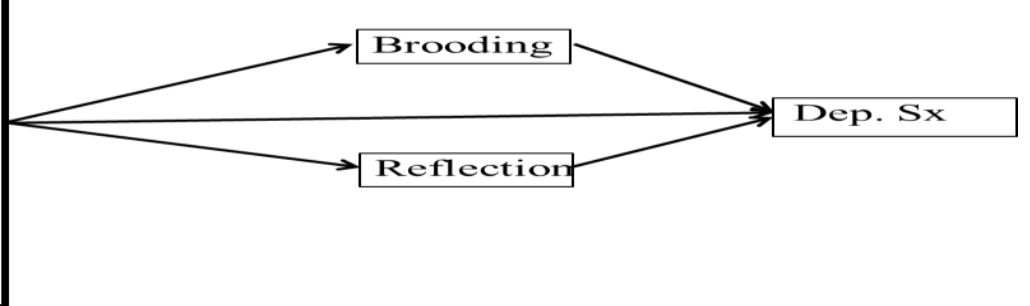

Model 6-Combined Integrative Model

Wave 1

Wave 2

Wave 3

\begin{tabular}{|l|l|}
\hline Globality & Dep. Sx \\
\hline Self & Brooding \\
\hline Conseq. & Reflection \\
\hline Stability & \\
\hline Internality & Globality x Reflection \\
\hline Globality x Brooding & Self x Reflection \\
\hline Solf x Brooding & Conseq. $x$ Reflection \\
\hline Stability x Brooding & Stability x Reflection \\
\hline Internality x Brooding & Internality x Reflection \\
\hline
\end{tabular}

Figure 1. Path diagram of Models 4 (moderation), 5 (mediation), and 6 (combined integrative). In the mediation and combined integrative models, all constructs in wave one have paths to constructs in waves two and three. For the sake of readability, this is represented by the three arrows coming from the thick box around the constructs at wave one. 


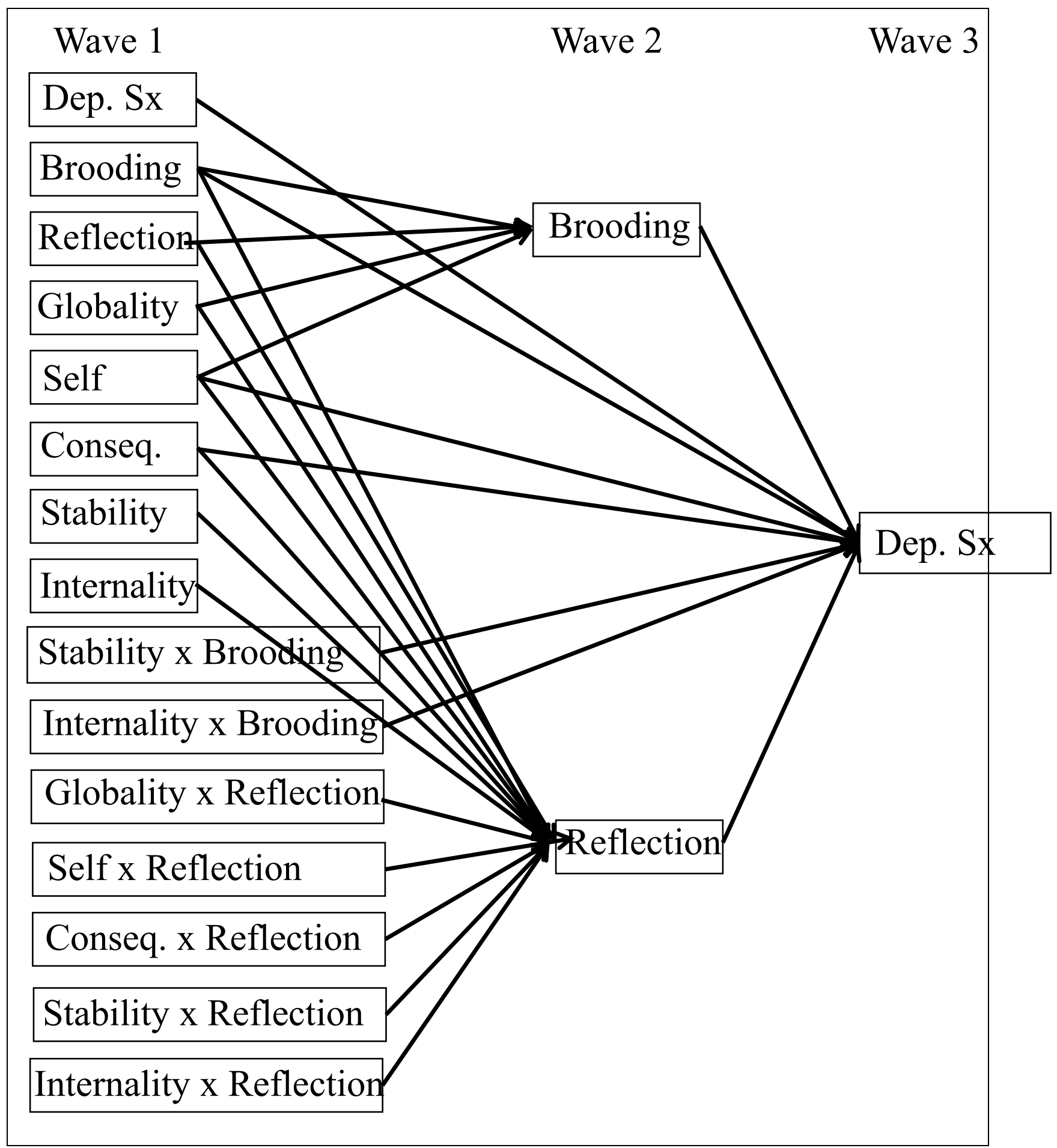

Figure 2. Path diagram of Model 7 (simplified combined integrative model). 